\title{
Calculating a District's Psychiatric Manpower
}

\author{
J. P. Watson, Professor of Psychiatry, United Medical and Dental Schools, Guy's Hospital, London SE1
}

The possibility that estimates of psychiatric manpower needs could be informed by knowledge of what doctors do and should do with their time was discussed in a previous paper. ${ }^{1}$ In this paper, we outline a procedure for calculating the psychiatric manpower needs of a Health District. The basic assumption is that the numbers of psychiatrists in a District ought to be sufficient to do the work that needs to be done. We suggest that this work should be assessed in terms of the amounts of doctor time required for specified activities.

\section{'Use of Time' norms}

We obtained estimates of how long particular activities should take from consultant colleagues including members of the College's Manpower Committee. Responses from 28 consultants were the basis for the results in Table I. We think these figures suggest a reasonable level of consensus about how long the activities mentioned might take in an average and acceptable, but not exceptional, service. Note that Table I indicates how much weekly time we think is needed for the activities specified from some doctor or other, not from a doctor of any particular status. So, on the basis of the entries in Table I we can calculate how much doctor time is needed to allow each acute in-patient to have one hour personally with a doctor, each new out-patient one hour, each long-stay patient 15 minutes, and so on.

Table I includes hospital-based elements of the psychiatric service. It excludes time to be allowed for travelling to community facilities, domiciliary visits, and prisons; experience is that travelling time is extremely variable from time to time and place to place, and may be considerable. It is important that it is estimated and allowed for locally to the extent that the service requires it. (Travelling also requires proper acknowledgement when clinical budgets are prepared, for it may be very expensive; but this is another matter).

Table I also makes no mention of time required for management and administration, and for academic activities, including teaching and research. Our respondents gave information about this; consultants appear to spend variable amounts of time in these ways. On average, consultants spent roughly four weekly hours in management or administration (over and above administrative tasks in immediate relation to patients, which is included under 'parapatient activity') but it is obviously common practice nowadays for most of the medical psychiatric administration to be done by only one or a minority of consultants in a District. Commensurate adjustments to clinical time should be made when this pattern is adopted. It seems reasonable however to allow four hours on average per consultant to management tasks.
This is one area where junior doctors and consultants' responsibilities differ somewhat. As regards academic activity, it seems reasonable also to allow four hours per doctor for this, even though content will vary between seniors and juniors. In detailed computations allowance must be made for seniors' training of juniors, when the same time appears on the timetable of all involved (e.g. consultant supervising one registrar for one hour is two doctor hours; consultant supervising four registrars for one hour each is eight hours, but together for one hour as a group is five hours).

TABLE I

Times required for doctor activities

\begin{tabular}{|c|c|c|}
\hline Activity & \multicolumn{2}{|c|}{ Required hours/week } \\
\hline A. Seeing patients personally & & $\begin{array}{c}\text { See } \\
\text { Notes }\end{array}$ \\
\hline In-patients: Acute & 1 & 1 \\
\hline Long stay & $\frac{1}{4}$ & 2 \\
\hline Out-patients: New & $i$ & 3 \\
\hline $\begin{array}{c}\text { Old } \\
\text { Day-patients: New }\end{array}$ & $\begin{array}{l}\frac{1}{2} \\
1\end{array}$ & 4 \\
\hline Old & $\frac{1}{4}$ & \\
\hline Others: Liaison referral & 1 & 5 \\
\hline Emergency & 1 & \\
\hline Domiciliary visit & 1 & \\
\hline Prison referral & 1 & \\
\hline B. 'Parapatient activity' & & 1 \\
\hline Acute in-patients & 1 & \\
\hline Long-stay in-patients & $1 / 8$ & \\
\hline C. Case conferences & & 6 \\
\hline Acute In-patients & 3 & \\
\hline Long-stay in-patients & 1 & \\
\hline Day hospital & 2 & \\
\hline
\end{tabular}

Notes to Table I

1. 'Acute in-patients' include newly admitted, and already admitted, patients. Computation of medical hours required to deal with in-patients is eased considerably if one hour is allocated for the occupant(s) of each bed, for an estimate can be made without needing to know the admission rate. This simplification will underestimate required weekly hours if the weekly admission rate exceeds the bed numbers. It seems likely that in many acute units a newly admitted patient is seen for more than one hour on arrival (in the first week) and for less than this in later weeks, regular weekly hourlong conversations between doctor and patient being a relatively infrequent part of acute in-patients' treatment programmes. The appropriateness of this practice perhaps requires research. Here, the assumption is that on average it should be possible for each in-patient to speak personally to a doctor for an hour a week. 
It should be noted also that 'parapatient' activity on behalf of acute in-patients requires as long again'.

2. 'Long-stay' patients. The estimate here is based on the aim that each long-stay hospital patient should see a doctor personally for a quarter of an hour weekly on average. Who the doctor should be is another matter. While it seems reasonable to suppose that "longstay' patients resettled in the community should also see a doctor regularly, this was not implied in respondent's comments and little is known about how much time doctors should spend personally seeing patients in community facilities.

3. There was near-unanimity that one hour is appropriately allowed for a new outpatient consultation.

4. It appears that 'old' out-patients include those whose consultations are relatively long (say $\frac{1}{2}-1$ hour) and others whose visits are brief (to $\frac{1}{2}$ hour). The appropriateness of continuing out-patient visits of either brief or longer duration seems an important topic for research. The allowance in the Table of $\frac{1}{2}$ hour is a rough 'average'.

5. As noted in the text, these figures refer to doctor-patient consultation time, excluding travelling where applicable. Characteristic of liaison work is that it can expand variably and considerably, to fill much increased time if this is made available. Hence often it is effectively limited by the limits of the time which can be made available rather than by consideration of which sort of patient are best dealt with by psychiatric referral.

6. Case conference times are per unit managed as a whole. If more than one doctor attends a conference, then appropriate medical hours should be assigned to the activity (e.g. one three hour conference with consultant, senior registrar and SHO utilises nine doctor hours).

\section{Calculating manpower needs}

Four steps are involved.

\section{Identify the components of the service}

In many instances, these may involve six headings, namely acute and long-stay inpatient beds; day hospital places; liaison service; out-patient clinics; and community facilities. As already noted, the content of 'community facilities' is extremely variable: group homes, hostels, and other residential facilities; resource centres or mental health advice centres; prison and special hospital visits; domiciliary visits; crisis intervention service; consultation to professionals in community agencies; and work in primary care settings, would all be included here.

\section{Specify the medical work to be done in each part of the} service

A. Acute in-patients. Let number of acute beds $=\mathrm{x}$. Assumption: each inpatient should see a doctor one hour a week, and will require one additional hour of parapatient activity. Therefore medical hours required $=2 \mathrm{x}$.

B. Long-stay inpatients. Let number of chronic beds $=y$. Assumption: each long stay patient should see a doctor $\frac{1}{4}$ hour a week on average, and will require an additional $\frac{1}{2}$ hour of parapatient activity.

Therefore medical hours required $=y / 2$.
C. Day hospitals. Let number of places $=\mathrm{z}$. Assumption: each new attender requires 1 hour on admission, and each continuing case requires $\frac{1}{4}$ hour weekly; for simplicity say $\frac{1}{2}$ hour on average weekly for each place, and an additional $\frac{1}{2}$ hour of parapatient activity. Therefore medical hours required $=\mathbf{z}$.

D. Liaison service. Let number of weekly referrals $=\mathbf{p}$. Assumption: each referral requires one hour on average. Hence number of medical hours required $=p$. (As implied previously, the size of $p$ can be set either by controlling the number of hours set aside for this service, or from the referral rate in the setting concerned.)

E. Out-patient service

The time required here depends critically on how much treatment is provided in addition to first consultations for opinion or assessment, for treatment implies repeat visits whose number and duration crucially affect the numbers of people needed to staff clinics. We assume that $m$ hours should be allocated for the assessment of $m$ new referrals each seen for one hour.

An additional two hours outpatient time would allow a patient two one hour sessions, or eight $\frac{1}{4}$ hour sessions; 10 one hour treatment sessions would obviously require 10 further hours. As discussed previously, ${ }^{1}$ serious psychological treatment programmes are rarely completed in less time than this. But at this stage we suggest between $2 \mathrm{~m}$ and $10 \mathrm{~m}$ hours (say $n$ hours) should be allocated for the treatment of the $\mathrm{m}$ patients whose assessment, as already noted, warrants $m$ hours. Therefore outpatient hours required $m+2 m \leqslant n \leqslant 10 m$.

F. Community facilities

As noted, Districts vary greatly in number of facilities existing and planned, their need for medical time, and associated travel time. Time involved 'in the community' can certainly be considerable, and should be computed specifically for each clearly defined activity. For convenience, an allowance of time for case conferences can also be made here. (This is an important variable; multidoctor or multiprofessional conferences can use up many doctor or professional staff hours. If these community facilities and conference activities $b_{1} \ldots b_{2}$ require $d_{1} \ldots d_{2}$ hours, then the total number of hours required is $\Sigma$ bd.

The total weekly medical hours $T$ required for all these activities is given by

$$
T=2 x+y / 2+z+p+m+2 m \leqslant n \leqslant 10 m)+\Sigma b d
$$

This equation includes no specific allowance for teaching, administration, and research. These should be allowed for by adding specified numbers of hours, perhaps conveniently derived from 'sessions'.

Two further comments may be made. The first is that activity is more easily modified in some of these categories than in others, and it is the more easily modified fields (liaison and outpatient services, and community facilities) in which activity changes when available hours are changed (typically nowadays reduced) by administrative fact motivated by financial or manpower considerations, unrelated to service needs. 
The other comment is that the equation is applicable to a sector in a District, or to a District comprising several sectors, in which case the approach provides information at new unit level relevant for budgeting and planning.

\section{Decide who should do this work}

Two decisions are involved here. The first concerns the possibility that some of the hours referred to herein as 'medical' ('psychiatrist') hours, may be required for activities which it may be agreed can be appropriately done by non-doctors. For instance, if it is agreed that liaison referrals, or some out-patients, should be seen by nurses or psychologists rather than doctors, then obviously medical hours need not be allocated to these activities. A good plan would be to apply the above calculations to 'team hours' rather than medical hours, estimating the hours to be committed managerially by professionals to a team with specified responsibilities. For example, a crisis intervention service might be planned to require two professionals on duty 9am until 5pm Monday to Friday and hence require (ignoring holidays etc) 80 weekly professional hours; planning would decide whether any or all of these hours should be contributed by doctors, with obviously different implications for manpower planning, finance, managerial arrangements, etc.

Having decided how many hours should be spent by doctors, it is necessary to decide how many of these should be spent by consultants and how many by junior doctors, trainee and non-trainee. Traditionally, some activitiese.g. seeing acute in-patients on admission-have usually been done by junior trainees, in the British system. Another traditional notion has been that initial out-patient visits should be to consultants. The present approach only proposes that specifiable numbers of medical hours must be available from doctors of some seniority or others for particular activities. In these terms, a trainee working in an acute unit could be replaced, hour for hour, by a consultant-but these would have to be additional consultant hours unless they are to be found at the expense of some other use of consultant time. Which clinical tasks can and should be done by doctors of which status seems another matter requiring urgent research. Current manpower policies unfortunately make exploration of these issues very difficult, because of attempts to allocate numbers of junior trainees and consultants separately from one another and on the basis of assessments of manpower needs unrelated to the work to be done. It is to be hoped that changed policies with respect to non-trainees may allow improved assessments of tasks which should best be done by non-trainee juniors. It is also worth noting that studies of parapatient activity would be helpful, to establish the extent to which these activities, customarily or traditionally done by doctors, may be done at least as well by secretaries, increasing the number of which might allow medical hours to be reduced without impairment of service.

\section{Assess future as well as current needs}

The starting point of this paper was the calculation of manpower required to deal with existing facilities. In the past new service developments-for instance the establishment of community mental health centres or of routine multidisciplinary domiciliary assessment in old age psychiatry - have typically been initiated using existing staff, including junior and senior doctors. Thus a new consultant psychogeriatrician might arrange for 'his' SHO to take part in community activities. This will however mean diversion of time from what the junior was doing before; with increasing controls on manpower numbers, opportunities for this kind of development will probably become fewer, and it seems essential to estimate medical need for all future service plans as additional to current establishment, unless all concerned agree that existing personnel should be redeployed.

One more important point about manpower requirements should be made. This is that current services have developed other than on the basis of need as assessed in terms of the frequency in the served population of specified mental disorders. In assessing a District's manpower needs a clear distinction should be made between the manpower needed to keep things going as they are, and that required to meet adequately all reasonable community needs for psychiatric service. Judgements about what are 'reasonable community needs' might begin with the most severe acute and chronic psychiatric illnesses, especially the schizophrenias and dementias, which have particular claim on specialist help.

If a previously unmet need is identified, and the District agrees that service should be provided, then it should expect that additional manpower will be required to do it. This should in turn include additional doctors if the jobs to be done can be specified and it is established that they cannot be done by staff already in post. There seems every reason to think that psychiatric service developments as officially encouraged by Health Departments and planned in many Health Districts will require increased medical as well as non-medical manpower. Hence continuing attempts by Manpower Committees to limit psychiatrist numbers seem uniquely inappropriate.

The extent to which a service meets specifiable population needs is an evaluation question to which, we suggest, every District should attempt to find the answer; for this adequate information systems will be essential.

\section{Implications for the Royal College of Psychiatrists}

The College's Manpower Committee has considered the matters discussed in this paper and advised Council that the use of 'norms' in planning of consultant psychiatrist numbers should be abandoned, and replaced by an approach such as that described here, which relates doctor numbers to the work to be done, acknowledging variations in local conditions and policies. 
Example

We complete this paper by summarising an attempt to apply this method to the author's Health District. Current facilities and responsibilities are summarised in Table II. doctors and nine consultants (WTE). It is indeed the case that the existing numbers of psychiatrists in the District can cope reasonably with existing workloads; hence the calculation indicates a reasonable correspondence between current

TABLE II

Facilities and staff needs in one Health District

\begin{tabular}{|c|c|}
\hline Facilities & $\begin{array}{l}\text { Doctor hours required } \\
\text { (see equation I) }\end{array}$ \\
\hline $\begin{array}{l}\text { A. Acute in-patients (number of beds }=x \text { ) } \\
\qquad x=170\end{array}$ & 340 \\
\hline $\begin{array}{l}\text { B. Chronic in-patients (number of beds }=y \text { ) } \\
\qquad y=350\end{array}$ & 175 \\
\hline $\begin{array}{l}\text { C. Day places (number }=\mathrm{z} \text { ) } \\
\qquad \mathrm{z}=\mathbf{8 0}\end{array}$ & 80 \\
\hline $\begin{array}{l}\text { D. Liaison (number of referrals }=p \text { ) } \\
\qquad p=30\end{array}$ & 30 \\
\hline $\begin{array}{l}\text { E. Out-patient clinics (see below) } \\
\text { Difficult to assess, but approx. } 40 \text { weekly doctor sessions of } 3 \\
\text { hours }\end{array}$ & 120 \\
\hline $\begin{array}{l}\text { F. Community facilities } \\
\text { Very difficult to assess, but not less than } 30 \text { hours (Mental Health } \\
\text { Advice Centre, etc) }\end{array}$ & $\frac{30}{775}$ \\
\hline
\end{tabular}

Note At the time this table was prepared, various temporary staff arrangements meant that it was not possible to obtain reliable figures for new and old out-patients throughout the District, and the length of the consultations. 'Numbers of clinics' gives a pointer to the amount of work involved.

On the bases discussed here, the District would need 775 weekly doctor hours to 'cover' its existing facilities (excluding out-of-hours and weekend cover). Note that this total does not include hours required for case conferences, or management, or academic activities, so underestimates required doctor time. Nevertheless, we can ask how many doctors are required to work $\mathbf{7 7 5}$ weekly hours.

On the basis of data reported earlier ${ }^{1}$ a busy junior general psychiatrist does not spend more than 20 hours in face to face contact with patients, including out-patients, spending time beyond this in 'parapatient activity', training and administration. (Some parapatient activity is allowed for in equation 1, but case conferences are not). If we assume one doctor can provide 20 hours weekly to the activities represented in equation 1 , then $775 \div 20=39$ doctors (WTE) would be needed to provide 775 hours. [If 'one doctor' $=24$ hours, the number of doctors needed becomes $775 \div 24=32$ (WTE).]

This should be compared with existing establishment. For the work under consideration this comprises 25 junior work load and numbers of available doctors. Local plans anticipate the expansion of psychiatric activity in the community, and it will be necessary to specify what current activity is to be replaced, or to obtain additional doctors to do the work. Manpower plans made regionally or centrally may propose reduced medical numbers for the District, in which case the service will mentally contract correspondingly. Colleagues are invited to apply the method in their own services.

To restate once again the most important point; potentially time consuming community developments may either require additional doctors if existing services are not to suffer, or need to be planned to be free of medical involvement. The time-consuming nature of psychiatry, medical and multiprofessional, must be understood by planners and managers as well as clinicians.

\section{REFERENCE}

'WATson, J. P. (1985) Psychiatric manpower and the work of the consultant. Bulletin of the Royal College of Psychiatrists, 9, 170-173. 